\title{
REPRESENTATION REQUIREMENTS OF AS-IS BUILDING INFORMATION MODELS GENERATED FROM LASER SCANNED POINT CLOUD DATA
}

\author{
$\underline{\text { Engin Burak Anil }}^{1 *}$, Burcu Akinci ${ }^{1}$, and Daniel Huber ${ }^{2}$ \\ ${ }^{1}$ Department of Civil and Environmental Engineering, Carnegie Mellon University, USA \\ ${ }^{2}$ Robotics Institute, Carnegie Mellon University, USA \\ *Corresponding author (eanil@andrew.cmu.edu)
}

\begin{abstract}
Obtaining and utilizing as-is information with 3D imaging technologies, such as laser scanners, during various phases of facility life-cycle is becoming common practice. Both during construction and facility operations information derived from laser scanner data can serve many purposes by providing accurate information about the conditions of the facilities at the time of the scanning. Currently, the information derived from point clouds is typically represented as building information models (BIMs). Despite the benefits of having accurate as-is BIMs, current BIM approaches and tools have limitations in representing as-is information. The reason is partly that current methods of as-is BIM generation are based on existing as-designed BIM generation and representation processes. We identified five main concepts that are unique to as-is BIMs and are not represented with existing BIMs. These characteristics are point density, noise, occlusions, model deviations, and the links between the points and building components.This paper investigates these unique characteristics of as-is conditions and discusses how representing them within a BIM can provide advantages to downstream users, such as enabling decisions based on more complete data than would otherwise be possible.
\end{abstract}

Keywords: As-is BIM, As-built BIM, As-designed BIM, Laser Scanner, Point Cloud

\section{INTRODUCTION}

Accurate as-is BIMs that are representative of facilities' existing conditions can help decision makers to base their decisions on actual and up-to-date information. Using laser scanners is becoming a common practice for collecting data for generating as-is BIMs by private owners and government agencies, due to its high accuracy, speed, and coverage[1,2]. However, current practices of as-is BIM generation from laser scanner data and representation approaches are based on existing as-designed modeling approaches. Laser scanner data and as-is BIMs generated from laser scanner data have unique characteristics, such as occlusions, point density, deviations, and measurement noise.Design modeling approaches were not developed for representing such imperfect properties of as-is conditions. Design objects are generally thought as being perfect, e.g., perfectly planar surfaces, which is not possible practically. The result is that there can be significant differences between the as-is data and the model derived from the data, and decisions made based on the as-is BIM may not be backed by actual data.The main aim of this paper is to discuss the unique characteristics of BIMs generated from laser scanners, so that these features can be incorporated into as-is BIMs and the benefits of having accurate and upto-date BIMs can be fully exploited.

\section{CHARACTERISTICS OF AS-IS BIMS GENERATED FROM LASER SCANNER DATA}

We have identified the following five concepts that are unique to as-is BIMs but are, as yet, not represented within as-is BIMs. :

- Point density: Point density is a measure of the average spacing between measurements on a surface in the environment. Point density affects the size of the smallest object that can be modeled.

- Uncertainty: Point clouds are the main source of information in the model generation procedure and are 


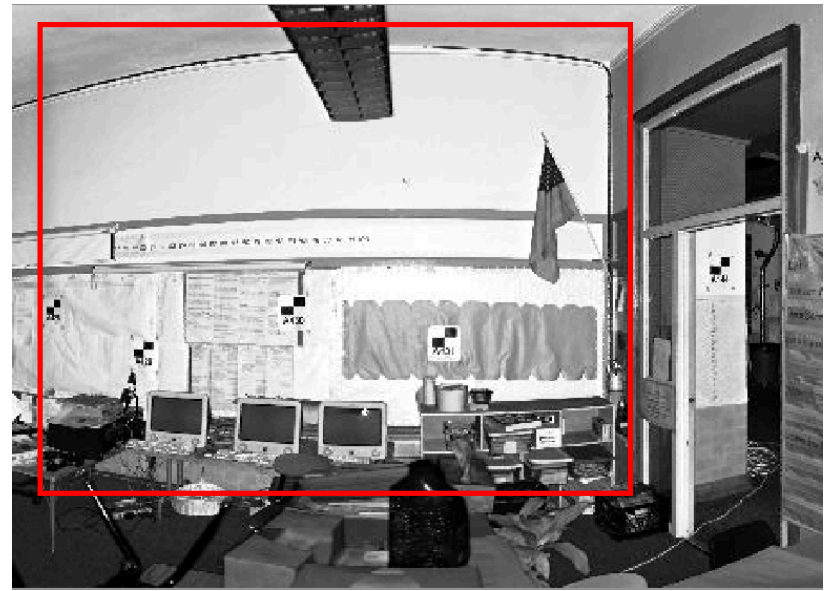

Figure 1. Reflectance image of the example wall surface (highlighted by the red box).

taken as the baseline. Therefore, uncertainty in the derived geometry will depend on the uncertainty in the measurements.

- Occlusions: Practically it is very difficult to observe every part of all relevant surfaces during scanning. Therefore, almost always there are objects in the models that are generated with incomplete data. Knowing these objects, or regions on the objects that are occluded can be important for accuracy-critical measurement tasks

- Deviations: Deviations of the model from the laser scan data can serve two major purposes. First, deviation information can be used to represent the surface geometries where accurately modeling the surfaces is not possible or practical. Secondly, deviation maps can be used to assess the quality of the as-is BIMs [1].

- Point clouds: Once a point cloud is converted into a BIM, the relationships of the BIM objects and the points that were used to generate the objects are lost. Maintaining the link between the BIM objects and point clouds can be useful for future reference.

\subsection{WALL SURFACE EXAMPLE}

We illustrate these factors unique to as-is BIMs using an example wall from a professionally generated as-is BIM. Figure 1 presents the reflectance image taken by the laser scanner. Figure 2 presents the BIM of the selected wall and the point cloud overlaid in the same environment. It can be seen from the figures that there is significant clutter in the environment as well as on the wall.

The following sub-sections will discuss characteristics of the as-is BIMs generated from point cloud data in further detail based on this practical example.

\subsection{COMPUTATION METHOD}

Currently, there is no commercial software that can automatically perform these computations on BIMs. Therefore, a custom workflow was developed.

Point density, noise, and deviations of point clouds relative to the model can be quantified by superimposing a $2 \mathrm{D}$ grid on the BIM surface and by calculating the corresponding properties on this grid. For example, point density can be computed as the number of points falling inside each grid cell.

Computations were performed using Open IFC Tools [3] to extract the surface geometry from the IFC file and a Matlab [4] script to perform the actual computations.

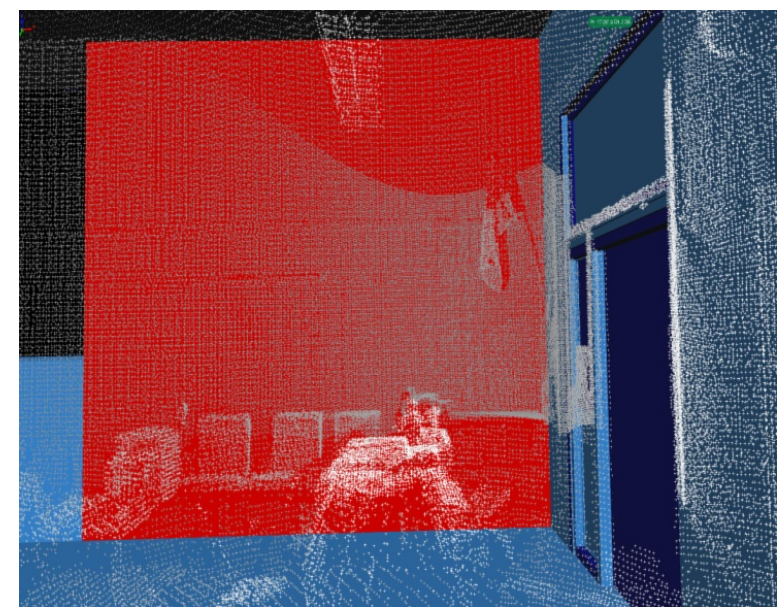

Figure 2. Example wall surface selected for point density, noise, and deviation computations. Point cloud and the $\mathrm{BIM}$ are visualized in the same environment. 


\subsection{POINT DENSITY}

In order to model an object, the spacing between the laser beams hitting a surface should be small enough so that the shape of the object or the details of the object can be observed. This property of the laser scan data can be quantified with the density of the scan data. Knowing the density of points that were used to model each object can help us assess whether the level of detail required in the project can be achieved by the collected data.

Different applications can have different level of detail requirements. For example, a BIM required for space usage planning and a BIM required for architectural renovation project have different point density requirements. While getting the general outline of the building spaces is the goal in the former case, centimeter or even millimeter accuracy can be required in the latter. GSA BIM Guidelines incorporate "Minimum Artifact Size" limits to enforce point densities based on the level of detail of the deliverables [5].

Point density varies depending on the scanning parameters over the model, i.e., resolution, number of scans seeing a particular surface, distance, and angle of incidence. Point density can be computed as the number of points falling in

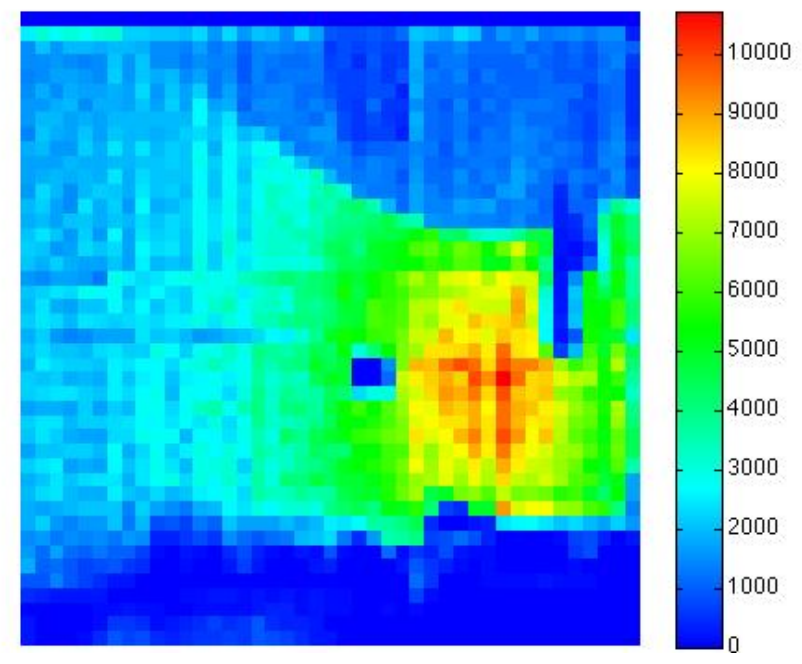

Figure 3. Point density on the example wall from Figure 1 (in points per square meter). the cells of a $2 \mathrm{D}$ grid, which is superimposed on the BIM surface. Figure 3 presents the point density map computed on the example wall surface from Figure 1. It can be seen from the figure that the point density is not constant over the surface. There is a highly dense region of points to the right of the center of the figure, and the density decreases towards the edges of the wall. The reason is in this particular example one of the scan locations is in front of the high-density region. As the range increases towards the edges of the walls and since the resolution parameters of the scanner are fixed, the point density decreases towards the edges. There are also regions without any data.

\subsection{UNCERTAINTY OF THE MEASUREMENT DATA}

Several factors lead to inaccuracy in point measurements and noise in the data, such as range, angle of incidence, surface reflectivity, and discontinuity edges on the target object. The inaccuracies can be quantified based on the point cloud, but in the current BIM approaches, measurement uncertainty cannot be represented. The result is that any dimensional measurement taken on the BIM will be regarded as exact, regardless of the quality of the underlying data. On the other hand, as stated in a report on measurement uncertainties by NIST, a measurement can only be regarded as complete if accompanied by a quantitative measure of its uncertainty [6].

Uncertainty can be computed as the standard deviation of the deviations of the points from the surface within each 2D grid cell superimposed on the wall surface. Figure 4 presents the uncertainty map of the points that fall on to the wall in Figure 1. The figure shows that uncertainty of the measurements on this wall range between $5 \mathrm{~mm}$ and $5 \mathrm{~cm}$. It can be said that reliability of the measurements taken on this surface varies, as the level of uncertainty at every location is different. In this particular example high-noise regions are due to reflections caused by the boards and shiny papers on the wall, computer screens, or mixed pixel 
effects around the edges of the computer screens and furniture seen in Figure 1.

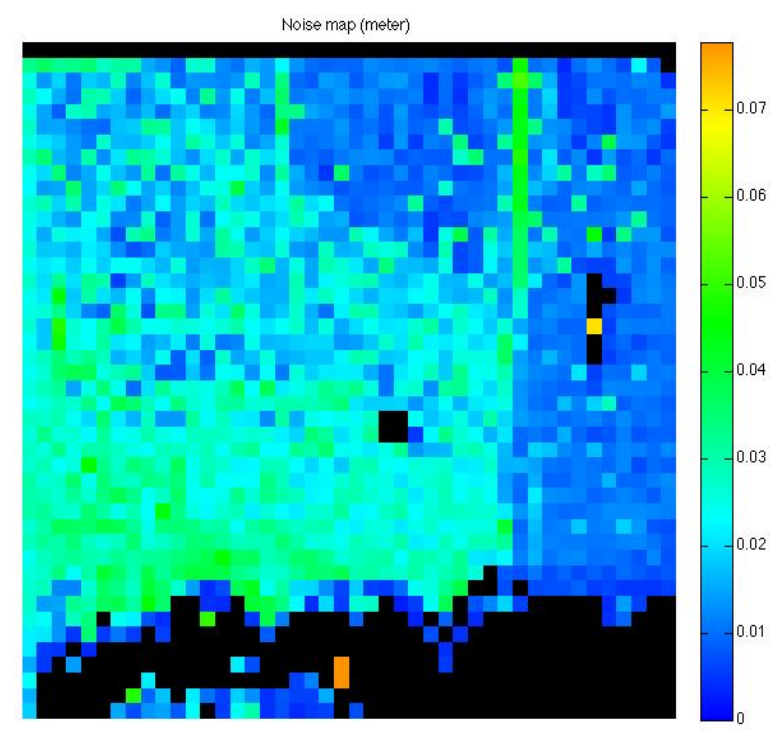

Figure 4. Uncertainty in the measurements quantified as the standard deviation of the deviations from the wall surface. Units are in meters.

\subsection{OCCLUSIONS}

Although coverage obtained with laser scanners is high, it is very difficult to obtain $100 \%$ coverage of the environment during data collection. The main reason is that the environment almost always contains self-occlusions. For example, back surfaces of the pipes or steel components, roof surfaces that are not visible from any other location, tight spaces such as closets, or ceiling surfaces behind the ceiling tiles are generally occluded. Almost always, there are equipment or furniture in the environment, which makes it impossible to obtain full coverage. It was found in a relevant study that $35 \%$ of the analyzed surfaces of a professionally generated BIM were occluded [7].

Partially or completely occluded objects are generally modeled by inferring dimensions and surface shapes. For example, a wall can be assumed to be continuous on the occluded regions. This means that almost always there are regions in the as-is BIMs that are unsupported by actual

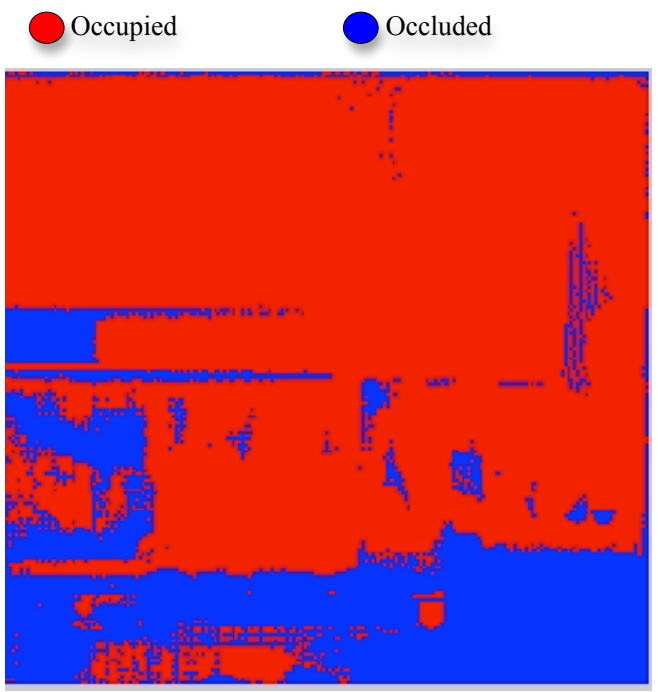

Figure 5 Occlusion map for the wall in Figure 1. Occluded regions are colored in blue.

data. The lack of occlusion information can result in potential problems in the usage of the BIM. For example, if the BIM is used for measuring dimensions for ordering prefabricated material for a renovation project at occluded locations which contain fixtures, equipment, or unmodeled components time delays can happen during production.

Knowing occlusion regions can be helpful for downstream users to guide them as to the proper spot to make measurements. Additionally, it is impossible to assess the reliability of the measurements if measurements are made at occluded regions.

Figure 5 illustrates occluded surfaces computed from the point clouds [8]. Being able to access occlusion information within the BIM can enhance the decision making based on as-is BIMs by showing which parts of the model are actually be backed up by data and which regions are not.

\subsection{MODEL GEOMETRY DEVIATIONS}

Geometry of real buildings is imperfect in the sense that, surfaces deviate from the assumed ideal geometries. While mathematical tools for representing model geometries are very rich in quantity and capabilities [9], generally 


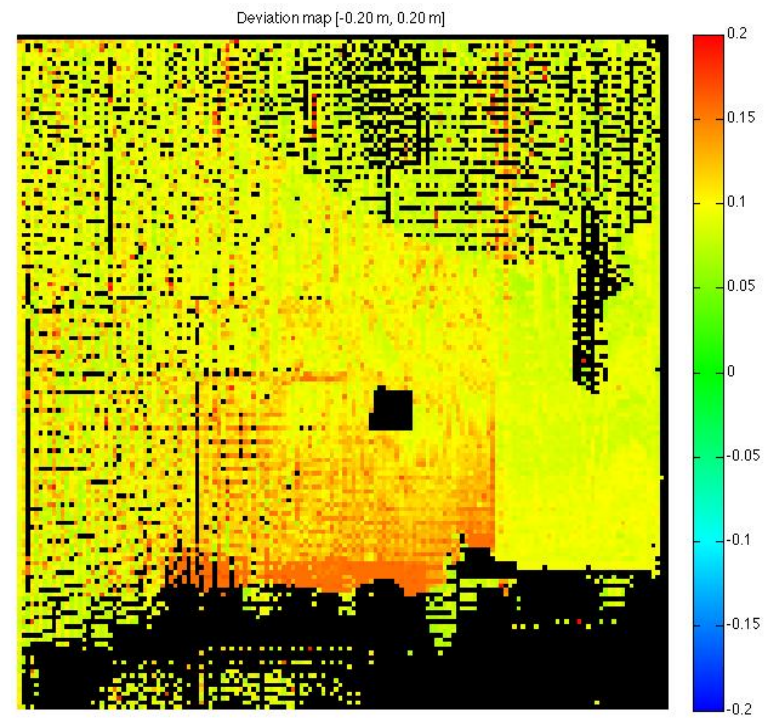

Figure 6. Deviations of the points to the wall surface. The upper and lower limits of the deviation map are selected to be -0.2 , and $0.2 \mathrm{~m}$. Points that are further than these limits are ignored.

simplifying assumptions dominate the as-is geometric modeling process. For example it is common to assume perfectly planar and plumb walls, orthogonal wall-to-wall intersections, and perfectly horizontal and flat floors.

Incorporating deviation information into as-is BIMs can allow simplifying assumptions for applications that need only an idealized representation without losing the true geometry. For example, a deformed surface can be represented with a planar patch and the deviation information, which is a finer representation of the surface geometry, can accompany the planar patch. Ultimately, the deviation information as well as uncertainty information can be incorporated into distance measurements performed on as-is BIMs.

Deviation analysis has also been presented as effective method of quality assessment of as-is BIMs [1]. By quantifying deviations of model surfaces from point clouds and analyzing the deviation maps for patterns of deviations, model quality issues can be identified. Modeling errors that can be identified with the deviation analysis method include, registration errors and calibration errors, missing components, incorrect position of components, and incorrect geometry of model objects.

Figure 6 presents a deviation map of the wall surface in Figure 1. The figure shows that while deviations from the plane range between $5 \mathrm{~cm}$ and $15 \mathrm{~cm}$, most of the wall surface is $10 \mathrm{~cm}$ away from the point cloud. This suggests that the wall location was modeled incorrectly in the modeling stage.

\subsection{POINT CLOUDS}

In the current practice, links between the model components and the individual points that were used to generate the components are not maintained. The result is that model generation procedure is one way, meaning that it is not possible to know which points were used to model certain components.

Linking the point clouds to the BIM in every case can be costly storage wise, but in certain cases maintaining this relationship can be useful. For example, if noise in a certain region is high and modeling is very difficult, the modeler can choose to maintain the point cloud only for that region within BIM for easy future reference.

\section{CONCLUSIONS}

In this paper, unique characteristics of as-is BIMs were investigated. The investigated characteristics in this paper are point density, uncertainty, deviations, and underlying point data. The current capabilities as-is BIM generation and representation do not support these characteristics. Moreover, current methods are based on existing asdesigned BIM approaches. The result is that assumptions made for as-designed models are assumed to be valid for as-is models, and discrepancies between the as-is model and the real geometry cannot be known in the as-is BIM utilization stage. As-is BIMs can be enhanced towards more realistic information representation by incorporating 
these characteristics.

There are two main challenges in incorporating these characteristics into as-is BIMs. First, it is not easy to quantify these properties with existing software tools. Second, there is no formalized method of representing this information with existing standards, such as IFC. To our best knowledge, existing software cannot compute these properties on a BIM. Although, certain software can perform deviation analysis on CAD objects, BIM to CAD conversion ultimately causes information loss [1].

The second challenge is developing formal representation methods for incorporating these characteristics into as-is BIMs. The biggest challenge here is that existing standards, such as IFC, were developed to represent design information, and it is not the primary interest of these standards to represent as-is conditions of buildings.

This paper focuses on the main unique characteristics of as-is BIM generated from point clouds and presents these characteristics through practical examples. The ultimate goal of this research is being able to represent these characteristics within BIMs so that more informed decisions can be made based on actual data and the real value of having accurate as-is BIMs can be exploited. The future work on this subject will focus on alternative representation methods of these unique characteristics of as-is BIMs with existing standards.

\section{ACKNOWLEDGEMENTS}

This material is based upon work supported, in part, by the National Science Foundation under Grant No. 0856558. Any opinions, findings, and conclusions or recommendations expressed in this material are those of the authors and do not necessarily reflect the views of the National Science Foundation. We thank Quantapoint, Inc., for providing experimental data.

\section{REFERENCES}

[1] E.B. Anil, P. Tang, B. Akinci, and D. Huber, "Assessment of quality of as-is building information models generated from point clouds using deviation analysis", SPIE IS\&T, 2011.

[2] G. Cheok, J.J. Filliben, and A.M. Lytle, "Guidelines for accepting 2D building plans", NIST Interagency/Internal Report (NISTIR) 7638,2009

[3] Open IFC Tools, www.openifctools.org, last accessed March 2011

[4] MathWorks Inc., Matlab 2010a.

[5] “GSA BIM Guide for 3D Imaging”, 2009, http:/www.gsa.gov/portal/content/102282

[6] B.N. Taylor and C.E. Kuyatt, TN 1297 - Guidelines for Evaluating and Expressing the Uncertainty of NIST Measurement Results, DIANE Publishing, 1994.

[7] D. Huber, B. Akinci, A. Adan, E. Anil, B. Okorn, and X. Xiong, "Methods for automatically modeling and representing as-built building information models," Proc. of NSF Engineering Research and Innovation Conference, Atlanta, Georgia: 2011.

[8] A. Adan and D. Huber, "3D Reconstruction of Interior Wall Surfaces Under Occlusion and Clutter," Proceedings of the British Machine Vision Conference (BMVC), 2010.

[9] P. Tang, D. Huber, B. Akinci, R. Lipman, and A. Lytle, "Automatic reconstruction of as-built building information models from laser-scanned point clouds: A review of related techniques," Automation in Construction, vol. 19, 2010, pp. 829-843. 